\title{
Torwards a more patient-centred healthcare system and effective services
}

\author{
Philip Michael Chircop \\ European Patients' Forum, Brussels, Belgium
}

The European Patients' Forum is a non-governmental, non-profit organisation set up in 2003 and based in Brussels. EPF is an umbrella body with currently 54 members - who are national coalitions of patients' organisations, and disease-specific patients' organisations active at European level. EPF's raison d'être is to contribute a strong and united patients' voice into EU-level policies that have an impact on patients' lives. EPF works with the EU institutions and agencies, as well as a range of other stakeholders, on a number of health-related policy areas and projects. EPF's vision is: high-quality, patient-centred, and equitable healthcare for all patients across the EU.

\section{The need for patient centred healthcare}

Healthcare systems have been traditionally set up to deal with acute care and episodic illnesses. To be able to face challenges (demographic, increase of patients with chronic illnesses, need for sustainability), and demands for higher quality and safety, healthcare systems need a fundamental change and be better geared toward meeting the needs of patients with chronic illnesses.

In recent consultations EPF members have highlighted that patients have to face obstacles relating to the organisation and delivery of health care (financial structures, productivity targets, attitudinal barriers,...). The result of this is that patients need to spend significant time and energy in fighting the system just to get the services they need. This lack of integration concerns all parts of the healthcare system: general practitioners, nurses, specialists, primary care institutions and hospitals, as well as community care.

EPF is convinced that the solution to meet these challenges while providing patients with the high quality, integrated care they need is to put in place genuinely patient centred care. Patient-centred care models have been shown to be cost-effective as well as increase patient satisfaction and often clinical outcomes. The benefits of integrated, patient-centred care are seen in terms of reducing avoidable

Correspondence: Philip Michael Chircop

CCopyright P. Chircop, 2013

Licensee PAGEPress, Italy

Thalassemia Reports 2013; 3(s1):e36

doi:10.4081/thal.2013.s1.e36

This article is distributed under the terms of the Creative Commons Attribution Noncommercial License (by-nc 3.0) which permits any noncommercial use, distribution, and reproduction in any medium, provided the original author(s) and source are credited.

Parts of this work were presented at the

"3rd Pan-European Conference on Haemoglobinopathies and Rare Anaemias", Limassol (Cyprus), 24-26 October 2012. hospitalisations, more effectively allocated healthcare resources, better quality care throughout the patient journey, and better informed, motivated and empowered patients.

\section{Patient involvement and empowerment}

Patient involvement is the cornerstone of patient centred healthcare. Healthcare decisions, at whatever level they are made, will ultimately affect patients' lives and the way they cope with their disease. From EPF perspective, patients are not only users of healthcare services, they have valuable experience in navigating the health system, and they have a key role to play in identifying issues in healthcare systems and solutions. It is absolutely critical to embed their perspectives, their expertise, and their unique experience as patients in healthcare decision making and in the innovation process. This applies at individual level, but also at collective level with the involvement of patients' organisations.

Patient involvement alone is not enough to achieve patient centred healthcare: Patients with chronic diseases (incl. families/informal carers) should be supported to participate proactively in the management of their condition. This can be done through empowerment strategies which include high quality information and health literacy. High quality, accessible information is a key pillar of patient safety and empowerment, and a key tool to enable meaningful patient involvement in their own healthcare.

To empower patients (and citizens), EPF believes there is a need for an overarching strategy at EU level on information to patients. That strategy should include a programmatic commitment to health literacy, and should be shaped with the input of patients. Health literacy means having the capacity to obtain, interpret and understand health information. People with low health literacy have poorer self-management skills.

\section{Enabling healthcare environment}

To empower patients and involve them meaningfully, an enabling healthcare environment is essential.

This includes ensuring that the principles of patient centred healthcare are implemented everywhere. Key avenues to achieve this include putting in place evaluation by patients of healthcare services, as well as to develop patient centred indicators for evaluating actions that relate to chronic diseases, and ensuring also collection of qualitative data with patient direct perspective.

Healthcare professionals have a key role to play. As they are the first trusted source of information for patients, and as such they play an essential role for patients' health literacy and empowerment. Informed and health literate patients need as a counterpart healthcare professionals that welcome patients' participation in shared decision making about their health and care, and that help create an enabling environment for a real dialogue and partnership approach. 


\section{The Chain of Trust}

A concrete example of how to promote patient-centred healthcare through effective trust-based patient and health professional organisations cooperation is the Chain of Trust project.

Led by the EPF and implemented through a sustainable partnership between the leading EU umbrella organisations representing end-users of telehealth services this project has undertaken a thorough assessment of the perspective of the main end users of telehealth services.

Key findings of this project:

- Chain of Trust tells us that while usefulness and advantages of telehealth are acknowledged by all user groups the large majority of patients and health professionals in Europe still have either no or very little experience with this type of services.

- Only 14\% of patients who responded to the project's online survey conducted in 2011 knew of the existence of telehealth services in their region.

- Only 6\% had used or was still using telehealth services at that time.

- While these figures are certainly not very encouraging given that eHealth and telehealth services have been around for more than 20 years, this project has amassed sufficient wealth of knowledge and evidence suggesting that there will be many more users if patients and health professionals were more aware of the opportunities presented by telehealth, and even more importantly, if these services were implemented starting from the needs of users and not the other way around.

- Telehealth also needs to be integrated in mainstream healthcare services as a complement and not as a replacement of conventional healthcare services. A reduction in physical meetings is not an option for patients and health professionals. Chain of Trust tells us that patients and health professionals perceive telehealth as largely beneficial when it helps eliminate unnecessary use of conventional healthcare services as a minimum level of face-to-face contacts is crucial to sustaining trust-based relationships among different end user groups. Key to fostering adoption of telehealth is to ensure users are fully confident in handling the additional responsibilities required by the integration of these types of services into care pathways.

- The Chain of Trust online survey revealed that while $92 \%$ of patients are quite keen to play a more active role in managing their own condition - which is a fundamental element inherent in all telehealth services, hence an essential precondition for acceptability of these type of services - only $60 \%$ of those who have never used telehealth would be willing to actually do so in the short-medium future. Less than $50 \%$ thinks they have the necessary skillset required to be able to handle the additional responsibilities arising from the adoption of telehealth.

- Concerning health professionals, the Chain of Trust online survey reveals that $70 \%$ of them, all three groups considered, who have never used telehealth before would be willing to use it in the short-medium future. Only $20 \%$ reported that the management in their workplace actually promotes the use of telehealth. $29 \%$ believe that their patients would be in the position to use telehealth services safely.

- A lot of work has to be done in terms of creating the necessary conditions for user acceptance. On the one hand, it is crucial to ensure that advancements in telehealth go hand in hand with concrete actions aimed at strengthening patients' health literacy. On the other hand, education and training for health professionals must also be provided. This needs to relate to the technology and to the new interaction and ways of communication between patients and professionals arising from the use of telehealth, as well as new roles and responsibilities.

- One of the objectives of the Chain of Trust project was to look into the various issues that are commonly deemed to represent major barriers to the adoption of telehealth services by patients and health professionals. These issues range from the lack of familiarity with IT equipment generating technology-related concerns especially among the elderly to threats to patient's privacy and confidentiality.

- Interestingly, this project found out that from a user-point of view all these may not necessarily be the real issues at stake when it comes to user acceptance. The concerns about threats to privacy and data confidentiality are overestimated. Likewise, lack of familiarity with the technology and IT equipment in general can have a negative impact on attitude and intention to use telehealth, but does not represent a major barrier to adoption as many technologyrelated concerns could be resolved through more user-friendly services and tools, integration of user requirements and effective education and training, and health literacy programmes.

- Chain of Trust tells us that many perceived barriers relating to technology disappear after a certain period of usage, especially if user requirements are effectively integrated in service design and implementation.

- The problem here is that people have traditionally looked at telehealth as a mere technological innovation in healthcare and have narrowly looked at the relationship between users and technology to identify barriers to acceptance. Elements such as human interaction, changing relationship between patients and health professionals have been largely side-lined, while there is sufficient evidence from this project suggesting that these factors are actually crucial to building confidence and trust.

\section{Conclusions}

To conclude, trust plays a key role in ensuring acceptance of telehealth among the different end user groups. There is no doubt that a key precondition for adopting telehealth is that both patients and professionals must trust the technology to be safe and effective. Building confidence in telehealth is not just about having trust in the technology itself, but also and perhaps even more importantly trust in other users.

Trust has traditionally been considered a foundation of effective health professional-patient relationships. Without trust patients may well not access healthcare services at all, let alone disclose all relevant information which is needed for health professionals to make informed decisions.

The shift towards more integrated care with the addition to telehealth as a complement to more conventional services and the changing role of patient from passive receiver to an active user who participates in decision-making is actually leading to greater inter-dependence between patients and health professionals.

The introduction of telehealth does, therefore, not lessen the need for trust in healthcare, but it actually demands more trust. Telehealth is about delivering healthcare services at a distance. This distance does not diminish the importance of the human dimension, but it actually makes it even more important since the reduction in physical contacts requires greater levels of mutual trust among different users. Patients need to trust health professionals, and at the same time health professionals need to trust and have confidence in their patients' ability to safely use telehealth.

As the developments in telehealth show, meaningful patient involvement and empowerment will become even more crucial and necessary with innovative form of care and cooperation between patient organisations and other stakeholder can help ensures that services are developed with the needs of the patients at the centre, and the input of the patients. This will in turn ensure that healthcare services are more effective in meeting users' need. 